\title{
Resiliencia y compromiso en estudiantes de Enfermería de la Universidad Nacional Jorge Basadre Grohmann en Tacna, Perú 2019
}

\begin{abstract}
Resilience and commitment in nursing students of the Jorge Basadre Grohmann National University in Tacna, Peru 2019
\end{abstract}

Resiliência e compromisso em estudantes de Enfermagem da Universidade Nacional Jorge Basadre Grohmann em Tacna, Peru 2019

\author{
Diana Yalpa Mojica ${ }^{\text {ab }}$ \\ Elena Cachicatari Vargas ${ }^{1 \mathrm{ac}}$ \\ Katia Flores Ledesma ${ }^{2,3 a d}$
}

(iD) https://orcid.org/0000-0002-8678-797X

iD https://orcid.org/0000-0002-9843-432X

iD https://orcid.org/0000-0002-9088-5820

\begin{abstract}
Resumen
Objetivo: Determinar la relación entre la resiliencia y compromiso en los estudiantes de Enfermería en Tacna, Perú. Material y métodos: Estudio no experimental, correlacional, de corte trasversal. La población estuvo conformada por 179 estudiantes de primero a cuarto año de la Escuela Profesional de Enfermería de la Universidad Nacional Jorge Basadre Grohmann en Tacna, durante el año 2019, a quienes se encuestó previo consentimiento informado, se aplicó dos cuestionarios validados. Para el análisis se utilizó el estadístico Chi cuadrado, con una confianza al $95 \%$. Resultados: Del total de estudiantes, el $88,8 \%$ fue de sexo femenino; el $57 \%$ de 19 a 21 años; el 60,3 \% expresó un nivel de compromiso alto y el 35,8 \% un nivel medio, siendo este compromiso alto por dedicación (69,3\%), vigor (52 \%) y absorción (55,3\%). El 54,7 \% presentó resiliencia de nivel alto y el $44,7 \%$, de nivel medio; siendo esta resiliencia alta por competencia personal $(96,1 \%)$, y por optimismo (60,3\%); el 44,7\% presentó un compromiso y resiliencia alta con una asociación estadísticamente significativa $(p<0,01)$, la resiliencia presentó asociación directa con todas las dimensiones del compromiso (dedicación, vigor y absorción) y el compromiso se asoció con todas las dimensiones de la resiliencia (competencia personal, confianza en el propio instinto, optimismo y relaciones) $(p<0,05)$. Conclusión: Existe relación significativa entre la resiliencia y el compromiso en estudiantes de Enfermería.
\end{abstract}

Palabras clave: resiliencia, compromiso, competencia para la vida

\section{Abstract}

Objective: To determine the relationship between resilience and commitment in nursing students in Tacna, Peru. Material and methods: Nonexperimental, correlational, cross-sectional study. The population was composed of 179 students from the first to fourth year of the Professional School of Nursing of the Jorge Basadre Grohmann National University in Tacna, during the 2019, who were surveyed with prior informed consent, two validated questionnaires were applied. For the analysis, the Chi square statistic was used, with a $95 \%$ confidence. Results: Of the total number of students, $88.8 \%$ were female; $57 \%$ were 19 to 21 year-old; $60.3 \%$ expressed a high level of

\footnotetext{
${ }^{1}$ Universidad Nacional Jorge Basadre Grohmann. Tacna, Perú

${ }^{2}$ EESPP Emilia Barcia Boniffatti. Lima, Perú

${ }^{3}$ Centro Internacional de Investigación y Desarrollo. Lima, Perú

${ }^{a}$ Licenciada en Enfermería

${ }^{\mathrm{b}}$ SERUMS

${ }^{\circ}$ Doctora en Enfermería

${ }^{\mathrm{d}}$ Doctora en Educación
} 
commitment and $35.8 \%$ a medium level, being this high commitment by dedication $(69.3 \%)$, vigor (52 \%) and absorption (55.3\%). 54.7\% showed high level resilience and $44.7 \%$ medium level resilience, with this resilience being high due to personal competence (96.1\%) and optimism (60.3\%); $44.7 \%$ showed high commitment and resilience with a statistically significant association $(p<0.01)$, resilience presented direct association with all dimensions of commitment (dedication, vigor and absorption) and commitment was associated with all dimensions of resilience (personal competence, self-confidence, optimism and relationships) $(p<0.05)$. Conclusion: There is a significant relationship between resilience and commitment in nursing students.

Keywords: resilience, commitment, competence for life

\section{Resumo}

Objetivo: Determinar a relação entre resiliência e compromisso em estudantes de enfermagem em Tacna, Peru. Material e métodos: Estudo não experimental, correlativo, de corte transversal. A população foi formada por 179 estudantes do primeiro ao quarto ano da Escola Profissional de Enfermagem da Universidade Nacional Jorge Basadre Grohmann em Tacna, durante o ano de 2019, quem foi entrevistado deu consentimento prévio, foram aplicados dois questionários validados. Para a análise utilizou-se o estatístico Chi quadrado, com uma confiança a $95 \%$. Resultados: Do total de estudantes, $88,8 \%$ foi do sexo feminino; $57 \%$ de 19 a 21 anos; $60,3 \%$ expressaram um nível de compromisso elevado e 35,8 \% um nível médio, sendo este compromisso elevado por dedicação $(69,3 \%)$, vigor (52 \%) e absorção (55,3 \%). 54,7 \% apresentaram resistência de alto nível e 44,7 \% de médio, sendo esta resistência elevada por competência pessoal $(96,1 \%)$ e por optimismo $(60,3 \%)$; $44,7 \%$ apresentaram um compromisso e uma elevada resiliência com uma associação estatisticamente significativa $(p<0,01)$, a resiliência apresentou associação direta com todas as dimensões do compromisso (dedicação, vigor e absorção) e o compromisso se associou com todas as dimensões da resiliência (competência pessoal, confiança no próprio instinto, otimismo e relações) $(p<0,05)$. Conclusão: Existe relação significativa entre a resiliência e o compromisso em estudantes de enfermagem.

Palavras-chave: resiliência, compromisso, competência para a vida

\section{Introducción}

El término resiliencia es relativamente nuevo. Para el Diccionario de la Real Academia Española, en el año 2016, la resiliencia es la "Capacidad de adaptación de un ser vivo frente a un agente perturbador o un estado o situación adversos". Sin embargo, etimológicamente, procede del latín saliere, el cual quiere decir "saltar hacia atrás, rebotar o surgir" y el prefijo "re" le añade al concepto de repetición."

El término compromiso deriva del latín compromissium. Este término se utiliza para especificar una obligación, un deber o incluso una promesa. Por lo tanto, el término compromiso también puede utilizarse para denotar responsabilidad por parte de aquellos que se comprometen a realizar un favor $o$ un acuerdo. $^{2}$
En el Perú, los jóvenes (con edades entre los 15 y los 29 años) constituyen ocho millones cuatrocientos cuarenta y un mil del total de los habitantes, de los cuales cuatro millones doscientos setenta y cinco mil son hombres y cuatro millones ciento sesenta y cinco mil mujeres. Al año 2016, se tiene que el $35,8 \%$ de jóvenes peruanos cuenta con educación superior. ${ }^{3}$

Una falta de resiliencia y compromiso frente a situaciones dolorosas, estresantes o difíciles puede afectar a los estudiantes universitarios a nivel académico y personal de manera negativa o positiva.

El presente estudio tiene como objetivo, determinar la relación que existe entre la resiliencia y el compromiso de los estudiantes de la Escuela Profesional de Enfermería, 
proceso de suma importancia para los futuros profesionales de salud, que necesitan identificar cómo se enfrentan a las diferentes adversidades.

\section{Material y métodos}

El presente estudio fue de enfoque cuantitativo, se asignó valores numéricos a los hallazgos, cuantificando los datos de las variables para hacer un análisis estadístico, lo cual permitió determinar el nivel de resiliencia y compromiso; de tipo no experimental, correlacional. ${ }^{4}$

La población de estudio estuvo conformada por 179 estudiantes universitarios de primero a cuarto año de estudios de la Escuela Profesional de Enfermería de la Universidad Nacional Jorge Basadre Grohmann ubicada en la ciudad de Tacna, Perú.

Se incluyó a estudiantes universitarios, que aceptaron participar en forma voluntaria del estudio, previo consentimiento informado. Se excluyeron a estudiantes que no completaron los instrumentos.

La validez de los instrumentos se realizó mediante evaluación de cinco expertos especializados en el tema de estudio; se consideró once indicadores de evaluación a juicio de expertos; la validación por criterio tuvo un valor de $72,96 \%$, lo cual significa una excelente validez.

Para la confiabilidad, se realizó una prueba piloto con estudiantes universitarios de la Escuela de Medicina de la misma universidad, previo consentimiento informado, y con Alpha de Cronbach de 87,40\% instrumento de resiliencia y 96,40 \% compromiso ambos con un Alpha de Cronbach de nivel muy alto.

En el aspecto ético se consideró los principios de autonomía, el consentimiento informado, la confidencialidad de los datos, principio de beneficencia y protección de grupos vulnerables.

\section{Resultados}

En la tabla 1, se describe la distribución porcentual para la edad de los estudiantes de
Enfermería, donde el mayor porcentaje fue de $57 \%$ entre 19 a 25 años, mientras que el menor porcentaje fue de $14 \%$ entre 22 a más años. En cuanto al sexo, el mayor porcentaje fue $88,8 \%$ femenino y el menor porcentaje fue $11,2 \%$ masculino. Respecto al año de estudios de los participantes, el $28,5 \%$ estuvo en primer año y el $22,3 \%$ en tercer año.

En la tabla 2, se muestra el nivel de compromiso de los estudiantes de Enfermería, donde el $60,3 \%$ tiene un nivel de compromiso alto, mientras que el menor porcentaje 3,9 \% tiene un nivel de compromiso bajo.

En la tabla 3, se observan las dimensiones del compromiso de los estudiantes de Enfermería, en la dimensión dedicación el 69,3 \% muestra un nivel alto, mientras que el $3,4 \%$, bajo; seguida por la dimensión de vigor, donde el $52 \%$ muestra un nivel alto y el $4,5 \%$, bajo; y la dimensión de absorción, donde el 55,3\% tiene un nivel alto y el $3,4 \%$, bajo.

En la tabla 4, se muestra el nivel de resiliencia de los estudiantes de Enfermería, donde el $54,7 \%$ tiene un nivel de resiliencia alto, mientras que el 0,6\% tiene un nivel de resiliencia bajo.

En la tabla 5, se muestran los resultados de las dimensiones de la resiliencia de los estudiantes de Enfermería, en la dimensión competencia personal, el 96,1 \% mostró un nivel alto, mientras que el 0,6\%, bajo; en la dimensión de confianza en el propio instinto, el $37,4 \%$ demostró un nivel alto, mientras que el $4,5 \%$, bajo; en la dimensión de optimismo el $60,3 \%$ obtuvo un nivel alto y el $1,1 \%$, bajo; finalmente en la dimensión de relaciones el $47,5 \%$ mostró un nivel alto, mientras que con $12,3 \%$, un nivel bajo.

En la tabla 6, se observa la relación entre la resiliencia y las dimensiones del compromiso de los estudiantes de Enfermería, donde el 69,3 \% muestra una relación alta entre la resiliencia y la dedicación, mientras que el 3,4 \%, una relación baja; seguida de la relación entre la resiliencia y el vigor, donde el $52 \%$ tiene una relación alta y el 4,5\%, baja; en cuanto a la relación entre la resiliencia y 
absorción, el 55,3 \% muestra una relación alta y el $3,4 \%$, una relación baja.

En la tabla 7, se muestra la relación entre las dimensiones de la resiliencia y el compromiso de los estudiantes de Enfermería, respecto a la relación entre competencia y compromiso, el 60,3\% tiene una relación alta, mientras que el 3,9 \% tiene una relación baja, con una relación significativa $(p<0,001)$; seguida de la relación entre confianza y compromiso, donde el 60,3 \% tiene una relación alta y el 3,9 \% tiene una relación baja, con una relación significativa $(p=0,001)$; en cuanto a la relación entre optimismo y compromiso, el 60,3 \% tiene una relación alta, mientras que el $3,9 \%$ tiene una relación baja, con una relación significativa $(p<0,001)$; finalmente, respecto a relaciones y compromiso, el $60,3 \%$ tiene una relación alta, mientras que el 3,9\% tiene una relación baja con una relación significativa $(p<0,001)$.

En la tabla 8 , se observa la relación entre la resiliencia y el compromiso de los estudiantes de Enfermería, donde el $60,3 \%$ tiene una relación alta, mientras que el menor porcentaje 3,9\% tiene una relación baja, con una relación significativa $(p<0,001)$.

\section{Tabla 1}

Características sociodemográficas de los estudiantes de la Escuela Profesional de Enfermería, Universidad Nacional Jorge Basadre Grohmann. Tacna, Perú 2019

\begin{tabular}{lcc}
\hline Sexo & $\mathrm{N}^{\circ}$ & $\%$ \\
\hline Masculino & 20 & 11,2 \\
Femenino & 159 & 88,8 \\
Total & 179 & 100 \\
\hline Edad & $\mathrm{N}^{\circ}$ & $\%$ \\
\hline De 16 a18 años & 52 & 29,1 \\
De 19 a 21 años & 102 & 57 \\
De 22 años a más & 25 & 14 \\
Total & 179 & 100 \\
\hline Año de estudios & $\mathrm{N}^{\circ}$ & $\%$ \\
\hline Primero & 51 & 28,5 \\
Segundo & 42 & 23,5 \\
Tercero & 40 & 22,3 \\
Cuarto & 46 & 25,7 \\
Total & 179 & 100 \\
\hline
\end{tabular}

\section{Tabla 2}

Niveles de compromiso en los estudiantes de la Escuela Profesional de Enfermería, Universidad Nacional Jorge Basadre Grohmann. Tacna, Perú 2019

\begin{tabular}{lcc}
\hline Compromiso & $\mathrm{N}^{\circ}$ & $\%$ \\
\hline Bajo & 7 & 3,9 \\
Medio & 64 & 35,8 \\
Alto & 108 & 60,3 \\
Total & 179 & 100 \\
\hline
\end{tabular}




\section{Tabla 3}

Dimensiones del compromiso en los estudiantes de la Escuela Profesional de Enfermería, Universidad Nacional Jorge Basadre Grohmann. Tacna, Perú 2019

\begin{tabular}{lcccccrcc}
\hline \multirow{2}{*}{$\begin{array}{l}\text { Dimensiones } \\
\text { compromiso }\end{array}$} & del & \multicolumn{2}{c}{ Bajo } & \multicolumn{2}{c}{ Medio } & \multicolumn{2}{c}{ Alto } & \multicolumn{2}{c}{ Total } \\
\cline { 2 - 10 } & $\mathrm{N}^{0}$ & $\%$ & $\mathrm{~N}^{\circ}$ & $\%$ & $\mathrm{~N}^{\circ}$ & \multicolumn{1}{c}{$\%$} & $\mathrm{~N}^{\circ}$ & $\%$ \\
\hline Dedicación & 6 & 3,4 & 49 & 27,4 & 124 & 69,3 & 179 & 100 \\
Vigor & 8 & 4,5 & 78 & 43,6 & 93 & 52 & 179 & 100 \\
Absorción & 6 & 3,4 & 74 & 41,3 & 99 & 55,3 & 179 & 100 \\
\hline
\end{tabular}

\section{Tabla 4}

Niveles de la resiliencia en los estudiantes de la Escuela Profesional de Enfermería, Universidad Nacional Jorge Basadre Grohmann. Tacna, Perú 2019

\begin{tabular}{ccc}
\hline Resiliencia & $\mathrm{N}^{\circ}$ & $\%$ \\
\hline Bajo & 1 & 0,6 \\
Medio & 80 & 44,7 \\
Alto & 98 & 54,7 \\
Total & 179 & 100 \\
\hline
\end{tabular}

\section{Tabla 5}

Dimensiones de la resiliencia en los estudiantes de la Escuela Profesional de Enfermería, Universidad Nacional Jorge Basadre Grohmann. Tacna, Perú 2019

\begin{tabular}{|c|c|c|c|c|c|c|c|c|c|}
\hline \multirow{2}{*}{$\begin{array}{l}\text { Dimensiones } \\
\text { resiliencia }\end{array}$} & \multirow[t]{2}{*}{ de } & \multicolumn{2}{|c|}{ Вајо } & \multicolumn{2}{|c|}{ Medio } & \multicolumn{2}{|c|}{ Alta } & \multicolumn{2}{|c|}{ Total } \\
\hline & & $\mathrm{N}^{\circ}$ & $\%$ & $\mathrm{~N}^{\circ}$ & $\%$ & $\mathrm{~N}^{\circ}$ & $\%$ & $\mathrm{~N}^{\circ}$ & $\%$ \\
\hline $\begin{array}{l}\text { Competencia } \\
\text { personal }\end{array}$ & & 1 & 0,6 & 6 & 3,4 & 172 & 96,1 & 179 & 100 \\
\hline $\begin{array}{l}\text { Confianza en } \\
\text { propio instinto }\end{array}$ & el & 8 & 4,5 & 104 & 58,1 & 67 & 37,4 & 179 & 100 \\
\hline Optimismo & & 2 & 1,1 & 69 & 38,5 & 108 & 60,3 & 179 & 100 \\
\hline Relaciones & & 22 & 12,3 & 72 & 40,2 & 85 & 47,5 & 179 & 100 \\
\hline
\end{tabular}

\section{Tabla 6}

Relación entre la resiliencia y las dimensiones del compromiso en los estudiantes de la Escuela Profesional de Enfermería, Universidad Nacional Jorge Basadre Grohmann. Tacna, Perú 2019

\begin{tabular}{|c|c|c|c|c|c|c|c|c|c|}
\hline \multicolumn{10}{|c|}{ Dedicación } \\
\hline \multirow{2}{*}{ Resiliencia } & \multicolumn{2}{|c|}{ Bajo } & \multicolumn{2}{|c|}{ Medio } & \multicolumn{2}{|c|}{ Alto } & \multicolumn{2}{|c|}{ Total } & \multirow{2}{*}{$\mathrm{CHI} \times 2$} \\
\hline & $\mathrm{N}^{\circ}$ & $\%$ & $\mathrm{~N}^{\circ}$ & $\%$ & $\mathrm{~N}^{\circ}$ & $\%$ & $\mathrm{~N}^{\circ}$ & $\%$ & \\
\hline Bajo & 0 & 0 & 1 & 0,6 & 0 & 0 & 1 & 0,6 & \multirow[b]{3}{*}{$P=0,000<0,05$} \\
\hline Medio & 6 & 3,4 & 31 & 17,3 & 43 & 24 & 80 & 44,7 & \\
\hline Alto & 0 & 0 & 17 & 9,5 & 81 & 45,3 & 98 & 54,7 & \\
\hline Total & 6 & 3,4 & 49 & 27,4 & 124 & 69,3 & 179 & 100 & \\
\hline
\end{tabular}




\begin{tabular}{|c|c|c|c|c|c|c|c|c|c|}
\hline \multicolumn{10}{|c|}{ Vigor } \\
\hline Bajo & 0 & 0 & 1 & 0,6 & 0 & 0 & 1 & 0,6 & \multirow{4}{*}{$P=0,000<0,05$} \\
\hline Medio & 8 & 4,5 & 47 & 26,3 & 25 & 14 & 80 & 44,7 & \\
\hline Alto & 0 & 0 & 30 & 16,8 & 68 & 38 & 98 & 54,7 & \\
\hline Total & 8 & 4,5 & 78 & 43,6 & 93 & 52 & 179 & 100 & \\
\hline \multicolumn{10}{|c|}{ Absorción } \\
\hline Bajo & 0 & 0 & 1 & 0,6 & 0 & 0 & 1 & 0,6 & \multirow{4}{*}{$P=0,000<0,05$} \\
\hline Medio & 6 & 3,4 & 48 & 26,8 & 26 & 14,5 & 80 & 44,7 & \\
\hline Alto & 0 & 0 & 25 & 14 & 73 & 40,8 & 98 & 54,7 & \\
\hline Total & 6 & 3,4 & 74 & 41,3 & 99 & 55,3 & 179 & 100 & \\
\hline
\end{tabular}

\section{Tabla 7}

Relación entre el compromiso y las dimensiones de la resiliencia en los estudiantes de la Escuela Profesional de Enfermería, Universidad Nacional Jorge Basadre Grohmann. Tacna, Perú 2019

\begin{tabular}{|c|c|c|c|c|c|c|c|c|c|c|c|}
\hline \multirow{3}{*}{\multicolumn{2}{|c|}{$\begin{array}{l}\text { Variable } \\
\text { independiente } \\
\text { dimensiones de } \\
\text { resiliencia }\end{array}$}} & \multirow{3}{*}{ la } & \multicolumn{6}{|c|}{$\begin{array}{c}\text { Variable dependiente } \\
\text { compromiso }\end{array}$} & \multirow{2}{*}{\multicolumn{2}{|c|}{ Total }} & \multirow{3}{*}{ Chi X2 } \\
\hline & & & \multicolumn{2}{|c|}{ Bajo } & \multicolumn{2}{|c|}{ Medio } & \multicolumn{2}{|c|}{ Alto } & & & \\
\hline & & & $\mathrm{N}^{\circ}$ & $\%$ & $\mathrm{~N}^{\circ}$ & $\%$ & $\mathrm{~N}^{\circ}$ & $\%$ & $\mathrm{~N}^{\circ}$ & $\%$ & \\
\hline \multirow{4}{*}{ 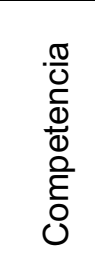 } & Bajo & & 0 & 0 & 1 & 0,6 & 0 & 0 & 1 & 0,6 & \multirow{4}{*}{$P=0,000<0,05$} \\
\hline & Medio & & 3 & 1,7 & 3 & 1,7 & 0 & 0 & 6 & 3,4 & \\
\hline & Alto & & 4 & 2,2 & 60 & 33,5 & 108 & 60,3 & 172 & 96,1 & \\
\hline & Total & & 7 & 3,9 & 64 & 35,8 & 108 & 60,3 & 179 & 100 & \\
\hline \multirow{4}{*}{ 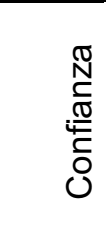 } & Bajo & & 0 & 0 & 7 & 3,9 & 1 & 0,6 & 8 & 4,5 & \multirow{4}{*}{$P=0,001<0,05$} \\
\hline & Medio & & 7 & 3,9 & 39 & 21,8 & 58 & 32,4 & 104 & 58,1 & \\
\hline & Alto & & 0 & 0 & 18 & 10,1 & 49 & 27,4 & 67 & 37,4 & \\
\hline & Total & & 7 & 3,9 & 64 & 35,8 & 108 & 60,3 & 179 & 100 & \\
\hline \multirow{4}{*}{ 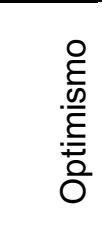 } & Bajo & & 0 & 0 & 2 & 1,1 & 0 & 0 & 2 & 1,1 & \multirow{4}{*}{$P=0,000<0,05$} \\
\hline & Medio & & 5 & 2,8 & 38 & 21,2 & 26 & 14,5 & 69 & 38,5 & \\
\hline & Alto & & 2 & 1,1 & 24 & 13,4 & 82 & 45,8 & 108 & 60,3 & \\
\hline & Total & & 7 & 3,9 & 64 & 35,8 & 108 & 60,3 & 179 & 100 & \\
\hline \multirow{4}{*}{ 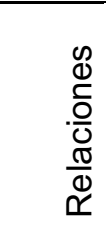 } & Bajo & & 0 & 0 & 15 & 8,4 & 7 & 3,9 & 22 & 12,3 & \multirow{4}{*}{$P=0,000<0,05$} \\
\hline & Medio & & 6 & 3,4 & 29 & 16,2 & 37 & 20,7 & 72 & 40,2 & \\
\hline & Alto & & 1 & 0,6 & 20 & 11,2 & 64 & 35,8 & 85 & 47,5 & \\
\hline & Total & & 7 & 3,9 & 64 & 35,8 & 108 & 60,3 & 179 & 100 & \\
\hline
\end{tabular}




\section{Tabla 8}

Relación entre la resiliencia y el compromiso en los estudiantes de la Escuela Profesional de Enfermería, Universidad Nacional Jorge Basadre Grohmann. Tacna, Perú 2019

\begin{tabular}{|c|c|c|c|c|c|c|c|c|}
\hline \multirow{3}{*}{ Resiliencia } & \multicolumn{6}{|c|}{ Compromiso } & \multirow{2}{*}{\multicolumn{2}{|c|}{ Total }} \\
\hline & \multicolumn{2}{|c|}{ Bajo } & \multicolumn{2}{|c|}{ Medio } & \multicolumn{2}{|c|}{ Alto } & & \\
\hline & $\mathrm{N}^{\circ}$ & $\%$ & $\mathrm{~N}^{\circ}$ & $\%$ & $\mathrm{~N}^{\circ}$ & $\%$ & $\mathrm{~N}^{\circ}$ & $\%$ \\
\hline Bajo & 0 & 0 & 1 & 0,6 & 0 & 0 & 1 & 0,6 \\
\hline Medio & 7 & 3,9 & 45 & 25,1 & 28 & 15,6 & 80 & 44,7 \\
\hline Alto & 0 & 0 & 18 & 10,1 & 80 & 44,7 & 98 & 54,7 \\
\hline Total & 7 & 3,9 & 64 & 35,8 & 108 & 60,3 & 179 & 100 \\
\hline
\end{tabular}

\section{Discusión}

La distribución porcentual del nivel de compromiso de los estudiantes de Enfermería, es de $60,3 \%$ en un nivel alto, mientras que de $3,9 \%$ en un nivel bajo. Además, se observa la distribución porcentual de las dimensiones del compromiso de los estudiantes de Enfermería, en cuanto a la dimensión dedicación, el 69,3\% es de nivel alto, mientras que el $3,4 \%$, de nivel bajo; seguida por la dimensión vigor, con el 52,0 $\%$ en un nivel alto y el 4,5\%, nivel bajo; y la dimensión absorción, con el 55,3 \% en un nivel alto y el $3,4 \%$ en un nivel bajo.

Según Maslach y Leiter definen al compromiso como un estado positivo, afectivo y emocional de completa plenitud que presenta la persona, y el cual tiene las características de vigor, dedicación, absorción y concentración para el trabajo y tareas. ${ }^{5}$

Schaufeli y Bakker ${ }^{6}$ hacen referencia a la voluntad y esfuerzo hacia las tareas y trabajos, y persistencia para afrontar dificultades. Dedicación: definida como el fuerte involucramiento y compromiso de la persona hacia su trabajo, que le van a permitir experimentar sensaciones de entusiasmo, inspiración y orgullo. Absorción: hace referencia al nivel de involucramiento y compromiso con el trabajo y tareas que se está realizando, donde el transcurso de las horas se da con rapidez y el hecho de abandonar lo que se está realizando, genera un sentimiento de molestia y desagrado.

Los resultados que engagement (compromiso) reportó Chávez ${ }^{7}$ para el nivel de engagement fueron altos en un $99 \%$ de su población estudiada, 40,1 \% más que en los estudiantes de Enfermería. Los resultados de Gonzales ${ }^{8}$ reportan que los estudiantes de la Carrera Profesional de Ingeniería Forestal de la Universidad Nacional San Antonio Abad de Cusco se ubican en el nivel alto en engagement referida a la absorción; en tanto, el $17,6 \%$ se ubica en el nivel medio y solamente el $2,2 \%$ en el nivel bajo.

De acuerdo a los resultados de Gonzales, ${ }^{8}$ el $80 \%$ de estudiantes tiene un nivel alto de engagement comparado con el presente trabajo, un 60,3 \% de los estudiantes de Enfermería también tiene un nivel alto de compromiso.

Considerando que el compromiso de los estudiantes podría deberse a la carrera escogida ya que se cuida a personas, existiendo momentos satisfactorios en función de las creencias y valores que se van desarrollando en el tiempo de la vida universitaria y que influirán de manera significativa en su formación como profesional. Se podría concluir que, los estudiantes universitarios de la Escuela Profesional de Enfermería presentan un alto nivel de compromiso con su carrera.

El nivel de resiliencia de los estudiantes de Enfermería, siendo el mayor porcentaje $54,7 \%$, es alto, frente al 0,6 \% que presenta un nivel de resiliencia bajo. Además, se observa la distribución porcentual de las dimensiones de la resiliencia de los estudiantes de Enfermería, en la dimensión competencia personal, el 96,1\% presenta un 
nivel alto, mientras que el 0,6 \% tiene un nivel bajo; respecto a la dimensión de confianza en el propio instinto, el $37,4 \%$ muestra un nivel alto, mientras que el 4,5\%, un nivel bajo; en la dimensión de optimismo, el 60,3\%, muestra un nivel alto y el $1,1 \%$, bajo; en la dimensión de relaciones, el $47,5 \%$ presenta un nivel alto y el $12,3 \%$, bajo.

En el ámbito de ciencias de la vida, la resiliencia expresa la adaptabilidad de los individuos o los grupos frente a los retos o amenazas y se ha definido como la capacidad para vivir, desarrollarse positivamente 0 superarse [...] frente al estrés o las adversidades que pueden normalmente ser causa de consecuencias negativas. ${ }^{9}$

Los resultados de Velásque ${ }^{10}$ indican que en su mayoría, con un $74,1 \%$, se encuentran en un nivel promedio, seguido de un $23 \%$ que se encuentra en un nivel alto, al compararlos con los resultados del presente trabajo se observa similitud ya que el $54,7 \%$ presenta un nivel de resiliencia alto. Concluyendo que, en ambos trabajos no existen niveles bajos de resiliencia en más de la mitad de estudiantes.

Los resultados de Ponte ${ }^{11}$ muestran que existe relación significativa, positiva y en grado medio, entre adaptación a la vida universitaria y resiliencia en los estudiantes, comparando con la presente investigación que nos dio un nivel alto de resiliencia con $54,7 \%$, podríamos afirmar que los estudiantes de la Escuela Profesional de Enfermería tendrían una buena adaptación con la carrera.

Los resultados de Rivas et al. ${ }^{12}$ indican que los estudiantes presentaron valores altos en la dimensión de competencia personal, de igual manera, en el actual trabajo también se indica que los valores de la dimensión de competencia personal en los estudiantes es de nivel alto $(96,1 \%)$.

Lo que indica que, a mayor resiliencia, mayor estado emocional y competencia personal, esto demuestra que se formarán profesionales de enfermería con niveles óptimos. Por lo que, este resultado es favorable para los estudiantes, ya que a lo largo de la profesión tendrán un antecedente positivo al momento de enfrentar las diversas situaciones negativas.

Respecto a la relación entre la resiliencia y las dimensiones del compromiso de los estudiantes de Enfermería. En resiliencia y dedicación, el 69,3\% muestra una relación alta y el 3,4\%, baja; en resiliencia y vigor, el $52 \%$ presenta una relación alta; en resiliencia y absorción, el 55,3\% muestra una relación alta y el 3,4 \%, baja. Además, se observa la relación entre las dimensiones de la resiliencia y el compromiso de los estudiantes de Enfermería; en competencia y compromiso, el $60,3 \%$ tiene una relación alta, mientras que el 3,9\% tiene una relación baja; en confianza y compromiso el 60,3\% tiene una relación alta, mientras que el 3,9 \% tiene una relación baja; en optimismo y compromiso el $60,3 \%$ tiene una relación alta, mientras que el 3,9\% tiene una relación baja; en relaciones y compromiso el $60,3 \%$ tiene una relación alta y el 3,9\% tiene una relación baja.

Pérez, ${ }^{13}$ en sus resultados evidenció que hay una correlación muy significativa y directa del constructo engagement con las dimensiones de la resiliencia, cada uno de ellos mostró un puntaje mayor alto. Estos resultados coinciden con el presente estudio ya que superan el $50 \%$ en todas las dimensiones. Además, encontró que en la dimensión absorción se mantiene un valor mayor a la mitad comparado con las otras dimensiones, esto es muy satisfactorio ya que toda la dimensión mantiene un puntaje significativo, estos datos difieren con el presente estudio ya que encontramos un nivel alto en relación de relación entre la resiliencia y la dimensión de la dedicación.

Lo que lleva a concluir que, los estudiantes universitarios presentan un nivel alto de resiliencia y las dimensiones de compromiso y viceversa, que irán fortaleciendo a lo largo de su carrera.

La relación de resiliencia y compromiso de los estudiantes de Enfermería, siendo el mayor porcentaje $60,3 \%$, es alta; frente al 3,9\% que es índice más bajo. 
Según Grotbert, la resiliencia es "la capacidad del ser humano para hacer frente a las adversidades de la vida", mientras que para Moreno, ${ }^{5}$ compromiso engloba las actitudes positivas de la persona o individuo hacia sus ocupaciones que incluye tanto el aspecto afectivo y cognitivo que se ve reflejado en todos los aspectos de la vida del ser humano.

De acuerdo a Cachicatari, ${ }^{14}$ existe una relación significativa entre el engagement y la resiliencia afirmando que el $73 \%$ poseen un nivel alto de relación, en comparación con el presente estudio existe similitud ya que un $60,3 \%$ también presenta una relación significativa entre el compromiso y resiliencia.

Asimismo, Pérez ${ }^{13}$ indica que dicha correlación es directa y con nivel de asociación a nivel fuerte. En síntesis, cuantos mayores sean los índices de resiliencia en los trabajadores, mayores también serán los índices en engagement. Este dato coincide con la presente investigación, afirmando que existe una relación significativa entre dichas variables.

\section{Referencias}

1. Martínez, I. y Vásquez, A. La resiliencia. La resiliencia invisible. Infancia, inclusión social y tutores de vida. Editorial Gedisa. 1ra. Ed. Barcelona; Pp 30-31(2006)

2. Real academia española. Compromiso $r$ e c u p e r a d o d e : https://dle.rae.es/compromiso

3. Gestión / Economía. Perú Tiene Casi 8.5 Millones De Jóvenes Pero ¿Cuántos Tienen Educación Superior? 2017 [Citada 2019 Dic 13]. [Alrededor De 1 Pantallas]. D i s p o i ble De sde: https://Gestion.Pe/Economia/Peru-8-5Millones-Jovenes-Educacion-Superior144111-Noticia/

4. Hernández Sampieri R. metodología de la investigación 6ta edición México: Mc. Graw Hill Education 2014 589p.

5. Martínez, I., Salanova M (2003). Niveles de Burnout y Engagement en estudiantes universitarios. Relación con el desempeño y desarrollo profesional. Revista de 47 Educación, volumen (330), pp. 361-384. Recuperado de: h t t p: // www.want.uji.es/wpcontent/uploads/2017/03/2003_Mart\%C3 \%ADnezSalanova.pdf

6. Schaufeli, W., \& Bakker, A. (2003). Utrecht Work Engagement Scale. Holanda: Utrecht University

7. Chávez D. Pando M., Aranda C. et al. Burnout y Work Engagement en Docentes Universitarios de Zacatecas, Ciencia y Trabajo [en línea] 2014 [17 Diciembre $2019] n^{\circ} 50$, disponible en: https://pdfs.semanticscholar.org/2c6c/c3d 86bf4ac9bb1c0b0beca841fc3437bd30d.p df?_ga=2.178257253.1598580972.15781 56802-1555470087.1578156802.

8. Gonzales D. Engagement en el estudio, inteligencia emocional y rendimiento en estudiantes de la carrera profesional de ingeniería forestal de la UNSAAC (tesis doctoral) Cuzco (2014).

9. Oriol A. Resiliencia Scielo 2012; volumen15 número 2: 77-78.

10. Velázquez P. Medida de la resiliencia en estudiantes universitarios de primer ciclo de la escuela profesional de administración y negocios internacionales de l a facultad de ciencias empresariales de la universidad alas peruanas. [maestría]. Universidad de Piura. Facultad de Ciencias de la Educación. Lima; 2017.

11. Ponte A. Adaptación a la vida universitaria y resiliencia en estudiantes de psicología de una universidad privada de Trujillo Perú 2017[Licenciado] universidad privada de Trujillo; Trujillo 2017

12. Rivas S.; Ordoñez J.; Mejia M. +et al. Resiliencia en Estudiantes de Medicina de Ia Universidad de Los Andes. SABER.ULA.VE, repositorio institucional de la universidad de los andes. 2017 [1712 - 19 ] d i s pon i b le e n : http://www.saber.ula.ve/handle/12345678 9/44358 
13. Pérez K. "Engagement y resiliencia en trabajadores de empresas contratistas de telecomunicaciones en Lima Norte 2017" [Licenciamiento] Lima, Perú Universidad Cesar Vallejo (2017
14. Cachicatari E. El Engagement Y La Resiliencia En La Enfermera Cuidadora Región - Tacna 2014. [tesis doctoral] UNSA Tacna; 2014.

\section{Correspondencia:}

ecachicatariv@unjbg.edu.pe
Fecha de recepción: 04/01/2021

Fecha de aceptación: 01/02/2021 